Research Article

\title{
Evaluation of Low-Carbon Competitiveness Based on a System Evaluation Method: A Case Study of Three Chinese Steel Companies
}

\author{
Jiang Yuguo $\mathbb{D}^{1},{ }^{1}$ Dennis Asante $\mathbb{D}^{\mathbb{D}},{ }^{2}$ Chen Dan $\mathbb{D}^{1},{ }^{1}$ and Zhang Jie $\mathbb{D}^{3}$ \\ ${ }^{1}$ School of Business, Sichuan Normal University, Chengdu, Sichuan 610101, China \\ ${ }^{2}$ School of Management and Economics, University of Electronic Science and Technology of China, Chengdu, \\ Sichuan 611731, China \\ ${ }^{3}$ College of Management Science, Chengdu University of Technology, Chengdu, Sichuan 610059, China
}

Correspondence should be addressed to Jiang Yuguo; daocaoren2007@126.com

Received 13 November 2020; Revised 14 March 2021; Accepted 24 March 2021; Published 10 April 2021

Academic Editor: Javier Martinez Torres

Copyright (C) 2021 Jiang Yuguo et al. This is an open access article distributed under the Creative Commons Attribution License, which permits unrestricted use, distribution, and reproduction in any medium, provided the original work is properly cited.

\begin{abstract}
Low-carbon competitiveness is an effective way for enterprises to remain relevant in the global green economy agenda. The lowcarbon footprints of firms are not only reflected in their carbon-tangible assets but also in carbon-intangible assets. Thus, carbonintangible assets also play a crucial role in firms' emission mitigation and environmental conservation capacity. Several forms of carbon-intangible assets may constitute an integrated system that shapes the low-carbon performance of enterprises. Therefore, firms' low-carbon competitiveness can be evaluated by studying the distribution of individual carbon-intangible resources and the composite shape of such assets. Based on this idea, this paper explored the dimensions through which carbon-intangible assets contribute to low-carbon competitiveness. By determining the location and distribution of each carbon-intangible asset in its value space, we proposed a multidimensional evaluation technique with three dimensions including realistic, sustainable, and inimitable value. Herein, the idea of value space of carbon-intangible assets has been contributed to the extant literature. Finally, to apply our evaluation index system, we selected three Chinese steel companies to serve as the research sample and evaluated their low-carbon performances. The main contribution of this study is the development and application of a novel multidimensional low-carbon evaluation method. This technique will not only help governments as well as the academics to effectively determine the low-carbon level of enterprises but also helps managers to fully grasp the trends of low-carbon competitiveness and thereby take some targeted measures to improve their firms' performance.
\end{abstract}

\section{Introduction}

The recent upsurge of climate anomalies has heightened a campaign for effective modes of greenhouse gases emission worldwide [1]. A myriad of natural disasters such as extreme weather conditions, famine due to instability in agricultural production, and increasing outbreak of infectious diseases are some of the adverse effects of unmitigated environmental pollution [2]. Several factors may account for these anomalies; however, the increasing industrial activities are considered key critical factors [3]. Hence, exploring environmentally sustainable development strategies has recently engaged the attention of both the international organizations and the academics alike. For instance, the United Nations Framework Convention on Climate change, the Kyoto Protocol, the Bali road map, Copenhagen Agreement, and the Paris Agreement are all geared towards finding solutions to the problems of climate change. Similarly, most scholars have explored several ways of solving the problem of greenhouse effect. Most of the existing environmental sustainability studies have focused on low-carbon production practices of industrial enterprises [4].

After the Copenhagen World Climate Conference, lower carbon concept has become an important component of developmental policies of the world economy. In recent years, the advancement of the processes required to achieve low-carbon emission rates has resulted in changes in the 
traditional competitiveness of enterprises [5]. This development led to the conceptualization of low-carbon competitiveness in the environmentalists' literature. Low-carbon competitiveness refers to the sustainable competitive advantage of enterprises through the adoption of low-carbon technologies and clean production methods to produce products and services with lower pollution and lower energy consumption [6].

With the advent of low-carbon economy, the concept of carbon emission is gradually outshining the traditional factors of production such as land, capital, and labor force as the major determinant of social and economic development. Thus, low-carbon competitiveness has become a key factor of sustainable competitive advantage of enterprises. An enviable hallmark of a company with a low-carbon competitive advantage is its ability to use energy more efficiently with lower levels of greenhouse gas emissions. Its carbon productivity (GDP output level per unit of carbon dioxide emissions) generally outperforms the industry benchmarks. Such feats normally induce policy rewards (such as tax incentives) and exemplary social reputation. In addition, a general reduction in corporate costs is one of the other several accrued benefits from low-carbon production practices.

Conversely, enterprises with poor low-carbon competitiveness, whose carbon productivity may be far lower than the industry benchmarks, normally incur high cost (high taxes and fines) and poor social image. Moreover, such firms normally grapple with regulatory policy constraints, which mostly force many out of business [7].

Low-carbon competitiveness can also be considered as an extension of traditional competitiveness, i.e., an "increment" of competitiveness gained by firms through various measures to reduce carbon intensity of their products and services to engender environmental sanity [5]. In the industrial settings, low pollution competitiveness can put enterprises at an advantageous position. Increasingly, most suppliers and customers are more inclined to choose lowcarbon enterprises. Therefore, enterprises with incremental competitiveness are likely to supplant the traditional dominant enterprises [8]. The most effective way for enterprises to thrive in the current era of economic globalization is to enhance their low-carbon competitiveness [9]. Excessive carbon emissions are spurned by the global community due to its negative effects on the environment. Thus, the low-carbon practices are encouraged strongly around the world. However, several enterprises have been struggling to improve their low-carbon competitiveness [5]. For such firms to effectively and strategically improve their carbon footprints, rigorous contextual scientific data will play a key role.

At present, literature related to low-carbon competitiveness is still relatively scanty, and the available data have primarily focused on three analytical levels-national, regional, and urban [5] - with very few studies involving enterprises [10,11]. The significant knowledge gap is to comprehensively analyze the factors that influence enterprises' low-carbon competitiveness and how to improve this competitiveness [6], even though some studies have detailed the macrofactors of enterprises low-carbon competitiveness. Nevertheless, only a few have gone deeper to explore the specific enterprise-related factors [8].

Meanwhile, modern researchers in this study domain have adopted the carbon asset principle proposed under the Kyoto Protocol to design their study frameworks [12]; for example, Han and Huang [13], Jiang et al. [5], Liu et al. [8], Jiang [11], and others have asserted that carbon assets principle should be central in any attempt to analyze human economic engagements and environmental conservation. In this regard, more investigations are needed on carbon assets involving low-carbon equipment, low-carbon technology, low-carbon culture construction, and low-carbon management at the level of industrial enterprises [14]. Such research designs should comprise both carbon-tangible assets and carbon-intangible assets. To achieve this, a more robust evaluation system capable of handling multiple indicators with several dimensions rather than the conventional evaluation approach is imperative.

The existing evaluation methods of single or multiple indicators are wrought with challenges yet to be addressed [15]; for instance, the contribution of a certain element of the evaluation object to the goal may be multidimensional; nonetheless, evaluation is a systematic work. Therefore, it is difficult to accurately, scientifically, and intuitively carry out multidimensional evaluation of the research objects using traditional evaluation methods [16]. At present, a scientific, practical, and targeted evaluation index system for lowcarbon competitiveness is in urgent need, and it is necessary to develop a multidimensional system evaluation method [17]. We, therefore, attempt to tackle the gaps alluded to in the current literature by proposing a novel multidimensional evaluation method based on carbon assets to assess selected Chinese steel companies' low-carbon competitiveness. To this end, this research offers the following invaluable contributions to literature:

(1) A three-dimensional evaluation space of low-carbon competitiveness of enterprises is constructed from the perspectives of realistic value, sustainable value, and inimitable value.

(2) A systematic evaluation of enterprises low-carbon performance based on the proposed model has been demonstrated.

(3) The empirical analysis in this study only not verifies the practicability and effectiveness of the proposed evaluation index system but also solves the problem of existing evaluation systems.

(4) The study findings offer insights into solving challenges associated with managerial decision-making on low-carbon management.

The remaining sections of the study are as follows: in Section 2, an overview of relevant literature is discussed. The evaluation space and the index system are provided in Section 3. In Section 4, the source of data and the process of evaluation are established. The discussion and implication are showed in Sections 5. Finally, Section 6 presents the study conclusions and limitations. 


\section{Literature Review}

With the development of society and technology, research on enterprise competitiveness has become increasingly diversified. In the context of the low-carbon era, the concept of low-carbon competitiveness has been derived. Low-carbon competitiveness is a major determinant of the survivability of enterprises in the future low-carbon economy. In addition, it describes the ability of enterprises to survive and develop better with the help of low-carbon economy [16]. A pioneering study in the field of low-carbon competitiveness research is Low-carbon Competitiveness of G20, in which the concept of low-carbon competitiveness, the ability of countries to create material prosperity for their people under low-carbon development approach in the future, was first introduced in a joint study published by the Australian Climate Research Institute and the British third-generation environmentalism organization. Wang et al. [6] define lowcarbon competitiveness as an organization's ability to continuously create value through low-carbon technologies, products, or services. Xin [18] reckoned that the competitiveness of a low-carbon economy is the ability to attract and optimize elements for wealth creation and improvement of national living standards under the constraints of carbon emission reduction targets. Although high carbon or low carbon will not change the nature of competition in any industry, in the process of competitiveness evolution, high carbon model leads to more unsustainable, and low-carbon model is bound to become the inevitable result of enterprise competition [5]. Enterprises usually possess tangible and intangible resources, which fuel competitive advantages; for instance, the scarce and nonreplicable, intangible resources play a decisive role in the competitiveness of enterprises [19]. The value of carbon-tangible assets can be accurately calculated and evaluated, and its contribution to the lowcarbon competitiveness of enterprises is embodied in a specific value $[16,20]$. Among low-carbon activities, although the value of carbon-intangible assets is not easy to determine, more difficult to quantify, and difficult to be recognized, they play a powerful role in driving enterprises to reduce carbon emission intensity and improve energy efficiency. This reflects the dynamic and sustainable competitiveness, such as enterprise low-carbon technology level, low-carbon human resource planning, low-carbon management level, and low-carbon organizational system [20]. This kind of carbon assets considerably controls the lowcarbon development of enterprises, comprehensively reflects the relative advantages of enterprises in low-carbon competition, and is an important source of enterprise lowcarbon competitiveness [5, 17].

The low-carbon competitive advantage of a company is seen when the company adopts low-carbon technology and cleaner production methods and, compared with its competitors, produces products and serves with low pollution, emissions, and energy consumption [10]. Therefore, in the era of low-carbon economy, a company's low-carbon competitive advantage is a key factor for its sustainable development. An enterprise with low-carbon competitiveness can use energy more efficiently and reduce energy consumption. Its carbon productivity will be higher than the industry benchmark value, thereby gaining policy bias and social reputation. At the same time, the cost of production of the enterprise can also be reduced. In contrast, companies that do not have low-carbon competitiveness may have carbon productivity far below the industry benchmark, coupled with high production costs and poor social outlook. They will be constrained by emission-restriction policies or even be forced out of the market [5]. The low-carbon competitiveness of an enterprise stems from the competitiveness theory, and research on the subject has mainly focused on the core competence view, resource-based view, and knowledge view [8]. The proponents of the core competence view believe that low-carbon competitiveness refers to the ability of an enterprise to reduce carbon emissions throughout the life cycle of their product(s). This specifically manifests in its low-carbon innovation ability, the formulation and execution of a low-carbon management system, and the ability to carry out clean production [21]. On the contrary, proponents of the resource-based view believe that the low-carbon competitiveness of an enterprise is dependent on its low-carbon resources, such as low-carbon technical resources, cultural resources, and management resources [22]. Knowledge-view advocates opine that lowcarbon knowledge is becoming a key resource and a major source of competitive advantage [23]. In an increasingly turbulent and complex market environment, a company's low-carbon competitive advantage depends not only on what it has but also what it knows [24].

At present, there is ample research on evaluation of enterprise competitiveness, but there are very few empirical studies on low-carbon competitiveness [5]. Fan and Jiang [17] adopted the entropy method to evaluate the low-carbon competitiveness of enterprises. Kuo et al. [25] also collected and calculated greenhouse gas emissions data based on the product life cycle and established a low-carbon evaluation model. Jiang et al. [5] discussed the importance of enterprise low-carbon competitiveness prediction and dynamic evaluation, improved the TOPSIS method, proposed a dynamic evaluation method, and conducted evaluation research on some enterprises. Xiao et al. [26] constructed an index system of enterprise low-carbon competitiveness and made an evaluation of some mechanical processing enterprises. After in-depth literature search, we found that research related to the systematic evaluation of low-carbon competitiveness has not yet appeared and needs to be further developed.

The evaluation of enterprise competitiveness needs scientific, reasonable, and practical evaluation methods. At present, academia mainly adopts a single comprehensive evaluation method, such as principal component analysis, analytic hierarchy process, TOPSIS method, fuzzy evaluation method, gray correlation evaluation method, neural network evaluation method, and data envelope method. Different evaluation methods have their own emphasis, and each has its own advantages and scope of application. When evaluating competitiveness, an appropriate method should be selected according to the specific purpose and objective of the evaluation [15]. The low-carbon competitiveness of an 
enterprise should be systematic; its value cannot be expressed by the simple addition of individual carbon-intangible asset values but should be formed by their systematical combination [16]. The value of any single carbonintangible asset, such as low-carbon intellectual property rights, low-carbon brand, and low-carbon management level, should be determined by the comprehensive capabilities of the enterprise [8]; for example, the research and development or the introduction of a low-carbon patent is an innovation activity. However, if the introduction of this patent does not improve the comprehensive capability or market development capability of the enterprise, then no matter how good the patent is or how high the market value is, it will be difficult for the patent to play its real role [27]. Therefore, to accurately and objectively evaluate the lowcarbon competitiveness of enterprises, it is necessary to design an evaluation space that reflects the value of carbonintangible assets, study the positioning and distribution of individual carbon-intangible assets in this space, and then analyze and measure this space system [16].

This article absorbs the thoughts and advantages of gray evaluation [28], proposes the research ideas of systematic evaluation, attempts to design three evaluation dimensions of "realistic, sustainable, and inimitable value," and establishes three-dimensional evaluation space [16]. We have positioned various types of carbon-intangible assets of enterprises in the evaluation space. The positioning patterns of various types of carbon-intangible assets of different enterprises have formed different low-carbon competitiveness. Finally, we used this method to evaluate the low-carbon competitiveness of three steel companies.

\section{Methodology}

3.1. Evaluation Space. Essentially, carbon-intangible assets are consistent with low-carbon competitiveness; i.e., they all reflect the low-carbon ability of enterprises [8]. The basis for measuring the low-carbon competitiveness of an enterprise is the quality and quantity of carbon-intangible assets [27]. However, unlike carbon-tangible assets, carbon-intangible assets are dependent on other assets of the enterprise. Identification of a particular carbon-intangible asset of an enterprise should be considered from a systemic perspective. In other words, the contribution of a certain carbon-intangible asset to an enterprise should be evaluated in the enterprise carbon asset system, rather than evaluating its value in isolation. The establishment of the value space of carbon-intangible assets needs to start from multiple dimensions. However, questions arise as to the most appropriate evaluation dimensions. The complexity of analysis increases exponentially with increasing dimension of analysis. The operation becomes more complicated, greatly increasing the workload and difficulty in evaluation, as well as its practicability [16]. Based on the above considerations, we appropriately merged and classified the characteristics of lowcarbon competitiveness and proposed a three-dimensional evaluation space.

First, we have to measure how much benefit carbonintangible assets can bring to the enterprise, i.e., their realistic value, which reflects the static low-carbon competitiveness of the company [16]. Therefore, obtaining realistic value is the ultimate goal of the low-carbon competitiveness of enterprises in the context of low-carbon economy. Only carbon-intangible assets that can be converted into realistic value have practical significance [27]. Carbon-intangible assets should also be able to maintain their ability or even continue to grow, in order to make them equally valuable in the future. This is to examine their sustainability [29]. In addition, a carbon-intangible asset with realistic value and sustainable value should not be easily imitated to maintain the competitive advantage of the company [20]. Based on the above analysis, we constructed a three-dimensional evaluation space for enterprise low-carbon competitiveness from three perspectives: realistic value, sustainable value, and inimitable value, as shown in Figure 1.

3.2. Positioning of Carbon-Intangible Assets. All types of carbon-intangible assets should have unique corresponding positions in the evaluation space. Determining the position of each type of carbon-intangible asset in the evaluation space is the first step in evaluating the low-carbon competitiveness of enterprises. The evaluation value of each type of carbon-intangible asset of an enterprise in each dimension of space forms a point in space. Therefore, the low-carbon contributions of various carbon-intangible assets form different points in the evaluation space, and the distribution of each point constitutes the total contribution of all carbonintangible assets of the enterprise and their overall value.

To evaluate the value of various carbon-intangible assets of a company in the evaluation space, i.e., to position them, the specific steps to follow include the following: (1) identify the carbon-intangible assets that reflect the lowcarbon competitiveness of the enterprise, select the main factors, and then determine the evaluation index system of low-carbon competitiveness. The evaluation index system includes the evaluation indices and weights of various carbon-intangible assets in various dimensions. The quality of the index system is directly related to the rationality and accuracy of the results, and thus, it needs to be selected by scientific methods. (2) Next, we need to collect the data required in the indicator system and spatially locate each carbon-intangible asset. For example, assume there are two companies, $\mathrm{A}$ and $\mathrm{B}$, and then, each carbon-intangible asset of company A (assuming there are 5 items, namely, A1-A5) will be evaluated according to three dimensions; each carbon-intangible asset of company $\mathrm{B}$ (B1-B5) can also be evaluated according to these three dimensions. Thus, the distribution structure of the carbon-intangible asset value of the two companies $A$ and $B$ in the evaluation space will be shown in Figure 2. (3) Finally, it is necessary to make a spatial positioning for the overall carbon-intangible assets of enterprises. By systematically integrating the value of carbon-intangible assets in Figure 2, for instance (such as vector summation), the low-carbon competitiveness of companies A and B can be obtained, as shown in Figure 3. 


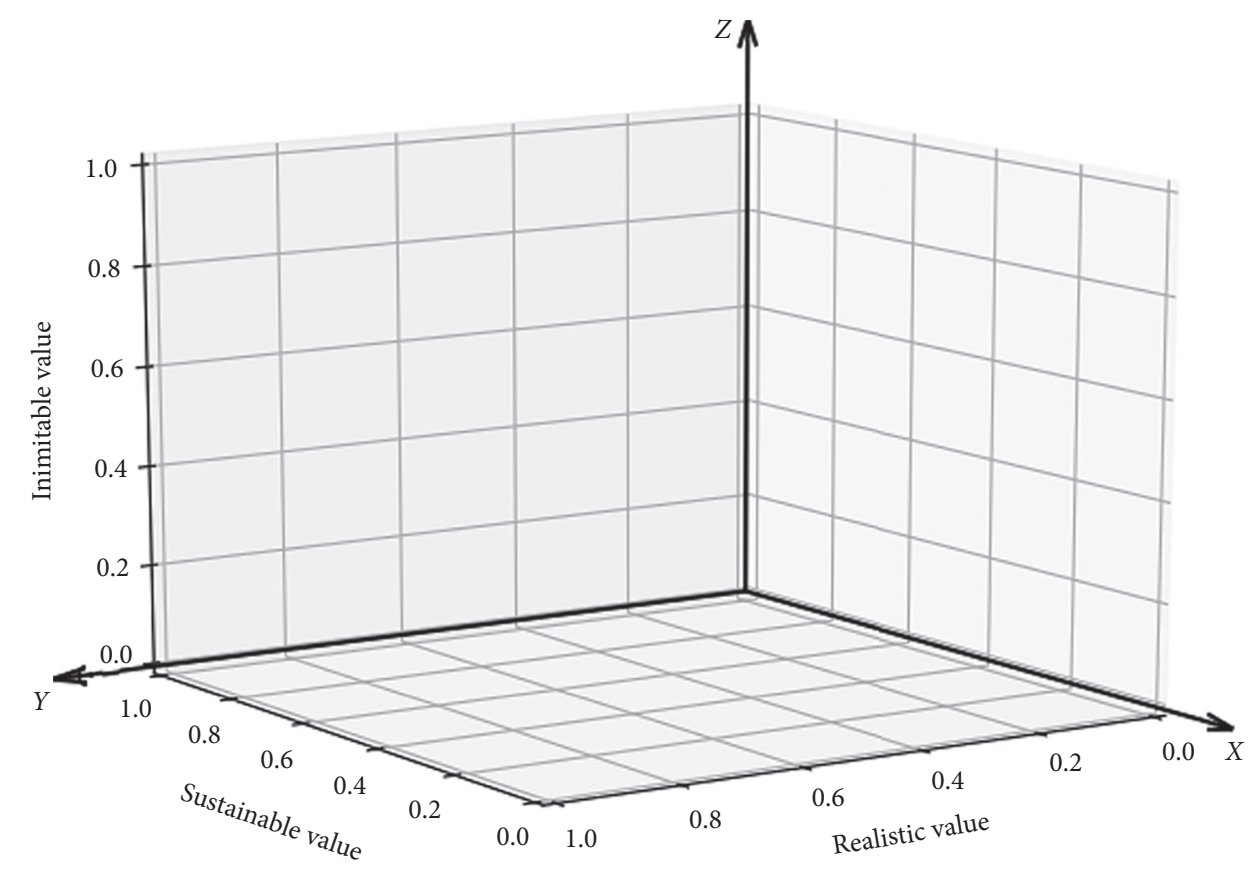

FIGURE 1: Evaluation space of low-carbon competitiveness of enterprises.

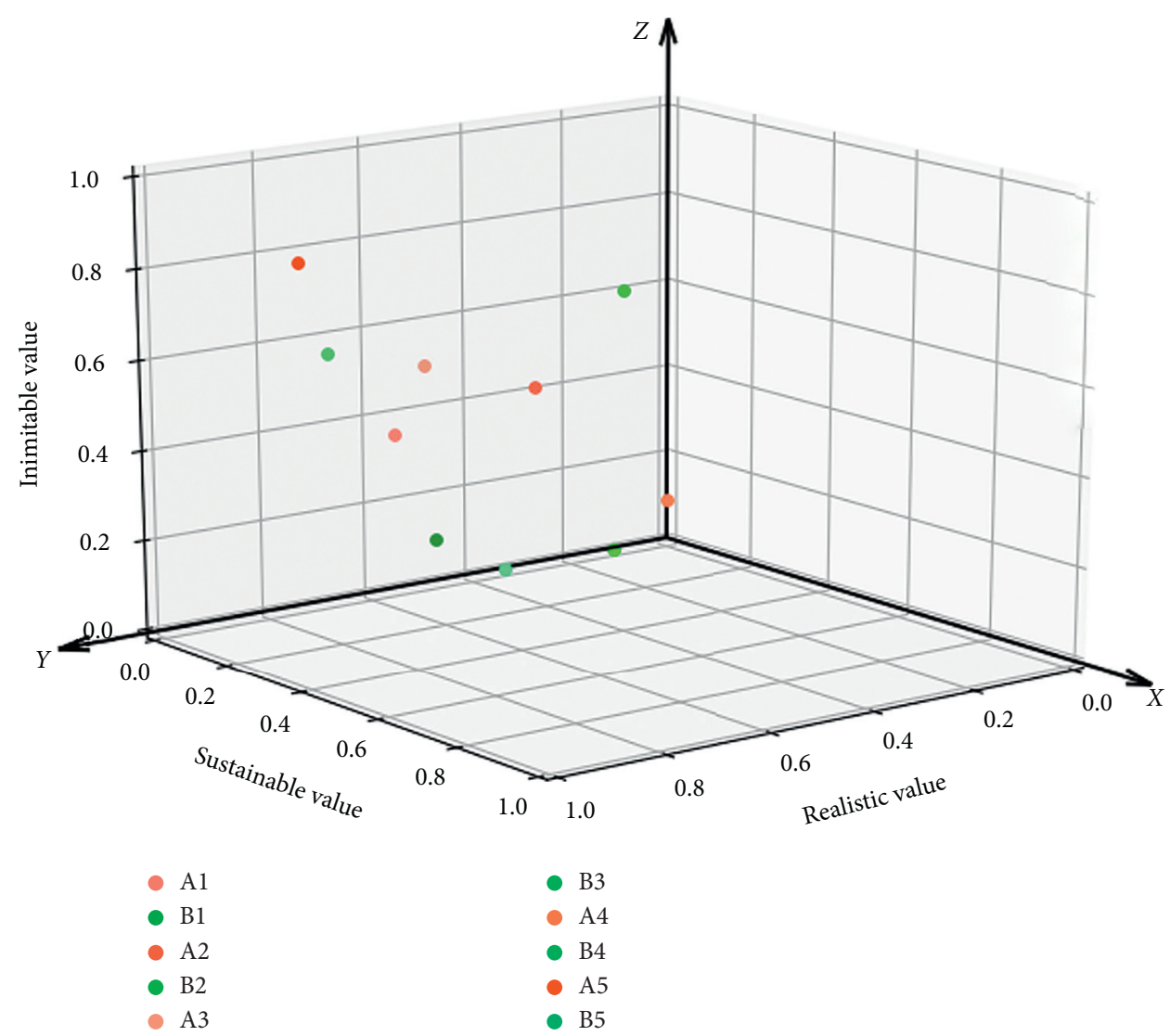

Figure 2: The value space structure of carbon-intangible assets of two companies, A and B.

3.3. Evaluation Index System. The evaluation index system determines the accuracy of the evaluation, and it is not a simple list of some indices. The indices should complement each other to form a system [15]. First, we must confirm the type of carbon-intangible assets, i.e., the core elements that reflect the low-carbon competitiveness 


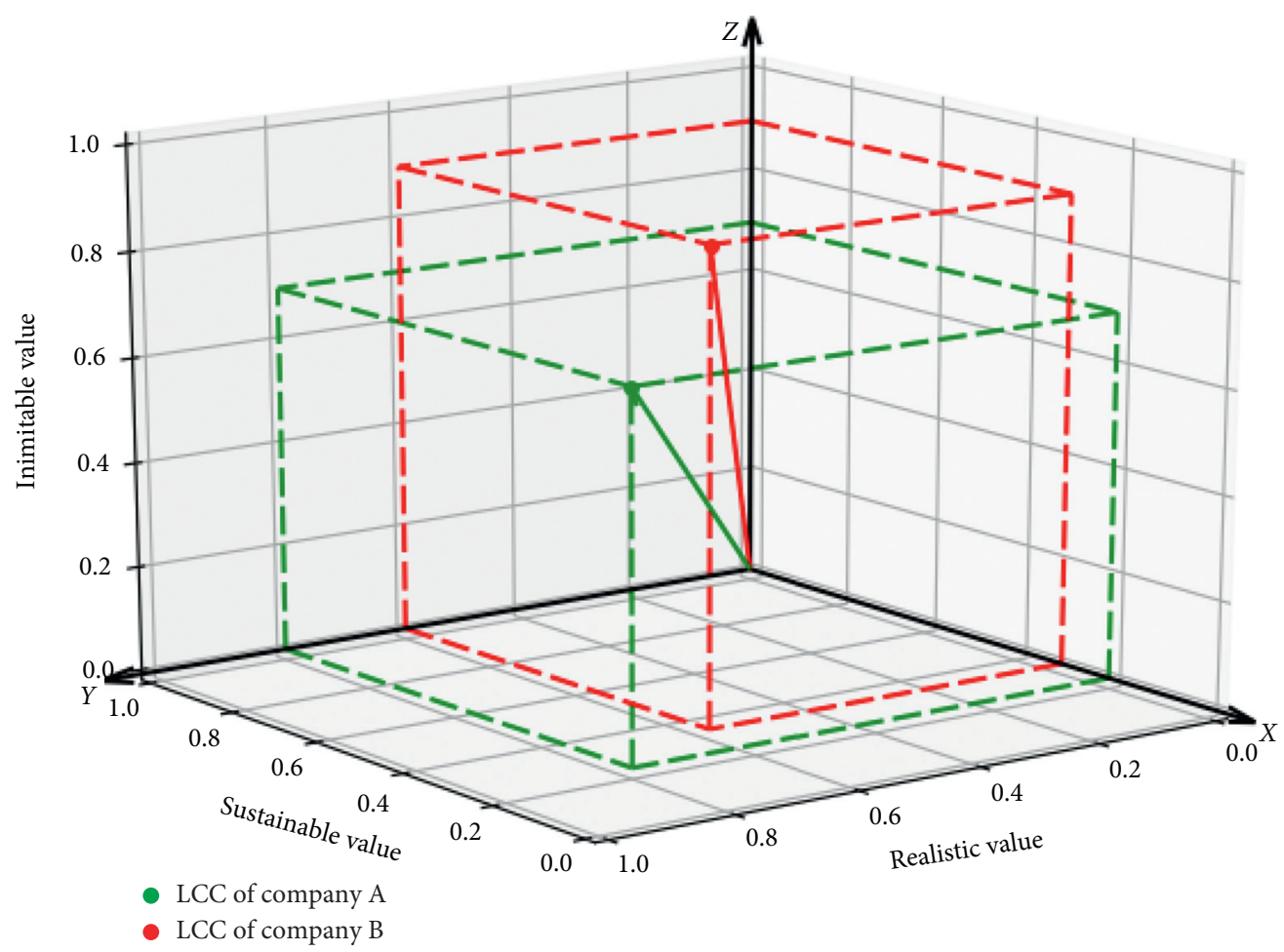

Figure 3: Low-carbon competitiveness of the two companies, A and B.

of enterprises. Jiang and Fan [30] described the factors that influence the low-carbon competitiveness from the perspective of carbon intangibles and proposed a model of these factors. Through empirical research, they proposed that low-carbon competitiveness mainly include five aspects: low-carbon human capital construction, technical level, cultural construction, market construction, and management level. We referred to the conclusions of Jiang and Fan [30] and selected these five elements for our research. In addition, in order to ensure the robustness of the evaluation index system, we divided the selection process into two processes: rough and careful selection. In the rough selection process, we mainly used literature search, brainstorming methods, and theoretical reasoning to collect all possible indicators in each evaluation dimension of various carbon-intangible assets. In the careful selection process, we used the Delphi method to optimize the index set. First, we selected experts, including professors from colleges and universities as well as business people (10 in total). A key criterion for selection is their familiarity with the theory or practice of the enterprise. Next, we followed the principle of systematicness and hierarchy, dynamism and stability, comprehensiveness and representativeness, qualitative analysis and quantitative calculation, scientificity, and feasibility to select indicators. Based on extensive selections, we constructed the index system with 5 elements and 31 indicators, as shown in Table 1. We mainly determined the weight based on the analytic hierarchy process, i.e., the scoring by 10 experts according to the industry status of the enterprise.

\section{Data Analysis and Results}

4.1. Evaluation Objects and Data Sources. In order to further investigate the low-carbon competitiveness of a company, we selected three Chinese steel companies to study and employed the evaluation method above to evaluate their low-carbon competitiveness. We obtained the consent of the enterprises and confirmed their willingness to participate in the study. Finally, we selected Shougang shares, Baosteel shares, and Angang shares as the research objects. The production size of each of the three companies is between 20 and 35 million tons, which is conducive to the comparison of enterprise data.

Governance structure and financial data were from the companies' annual reports, and the number of patents and other data were from the companies' official websites and the Intellectual Property Office. Overall, data were gathered between 2016 and 2018. For data with a large gap, the average value was taken. Part of the data also came from the research of Fan and Jiang [17]. The scores of qualitative indicators were mainly derived from the scores of 10 low-carbon experts, and the evaluation value of each enterprise was determined by the fuzzy evaluation method. We normalized the data to achieve standardization. Limited to the length of the paper, the following is the detailed evaluation of the three dimensions of human resources, and the other four elements can be analogized.

4.2. Evaluation Ideas. Suppose that the evaluation object $P$ has $m$ evaluation factors, the factor set is $U=\left\{u_{1}, u_{2}\right.$, $\left.\ldots, u_{m}\right\}$, and the evaluation level set is $V=\left\{v_{1}, v_{2}, \ldots, v_{m}\right\}$. 
TABLE 1: Evaluation index system of enterprise low-carbon competitiveness.

\begin{tabular}{|c|c|c|c|c|}
\hline \multirow{2}{*}{ Evaluation item } & \multirow{2}{*}{ Dimensionality } & \multicolumn{2}{|l|}{ Evaluation index } & \multirow{2}{*}{ Ability weight } \\
\hline & & Index & Weight & \\
\hline \multirow{6}{*}{ Human resources } & \multirow{2}{*}{ Realistic value } & Profit margin per capita & 0.60 & \multirow{6}{*}{0.13} \\
\hline & & Level of human resource development & 0.40 & \\
\hline & & Cost of low-carbon human resource management & 0.50 & \\
\hline & Sustainable value & Learning level of employees & 0.50 & \\
\hline & \multirow{2}{*}{ Inimitable value } & Low-carbon level of human resource management & 0.60 & \\
\hline & & Low-carbon quality index of employees & 0.40 & \\
\hline \multirow{7}{*}{ Techniques level } & \multirow{2}{*}{ Realistic value } & Total value of low-carbon intellectual property & 0.40 & \multirow{7}{*}{0.26} \\
\hline & & Sales proportion of low-carbon products & 0.60 & \\
\hline & & Proportion of low-carbon R\&D investment & 0.20 & \\
\hline & Sustainable value & Proportion of low-carbon R\&D personnel & 0.40 & \\
\hline & \multirow{3}{*}{ Inimitable value } & The number of low-carbon patents owned by the company & 0.40 & \\
\hline & & Success rate of low-carbon product development & 0.60 & \\
\hline & & Protection capabilities of low-carbon intellectual property & 0.40 & \\
\hline \multirow{6}{*}{ Cultural construction } & \multirow[b]{2}{*}{ Realistic value } & Employee satisfaction index & 0.30 & \multirow{6}{*}{0.16} \\
\hline & & Recognition of corporate low-carbon image & 0.70 & \\
\hline & & Investment level of enterprise low-carbon culture & 0.60 & \\
\hline & Sustainable value & Matching of corporate identity system (CIS) and enterprises & 0.40 & \\
\hline & \multirow{2}{*}{ Inimitable value } & Low-carbon awareness index of employees & 0.50 & \\
\hline & & Capacity of organizational learning & 0.50 & \\
\hline \multirow{6}{*}{ Market construction } & \multirow{2}{*}{ Realistic value } & Share of the low-carbon market & 0.40 & \multirow{6}{*}{0.22} \\
\hline & & Growth rate of operating income from low-carbon products & 0.60 & \\
\hline & & Plan of low-carbon marketing & 0.70 & \\
\hline & Sustainable value & Ratio of sales expenses to main income & 0.30 & \\
\hline & \multirow{2}{*}{ Inimitable value } & Low-carbon user satisfaction index & 0.60 & \\
\hline & & Perfection of marketing network & 0.40 & \\
\hline \multirow{6}{*}{ Level of management } & \multirow{2}{*}{ Realistic value } & Comprehensive energy consumption per unit output & 0.70 & \multirow{6}{*}{0.23} \\
\hline & & The effective rate of utilization of the energy & 0.30 & \\
\hline & alue & Growth rate of low-carbon fixed asset investment & 0.40 & \\
\hline & Sustainable value & Flexibility of organizational structure & 0.60 & \\
\hline & \multirow{2}{*}{ Inimitable value } & Level of low-carbon planning & 0.50 & \\
\hline & & Advancement of production technology & 0.50 & \\
\hline
\end{tabular}

Each factor in $U$ is fuzzy judged according to the rank index in the evaluation level set, and a judgment matrix $R$ is obtained, i.e., $R=\left(r_{i j}\right)_{n \times m}$, where $r_{i j}$ is the membership degree of $u_{i}$ with respect to $v_{j}$. $(U, V, R)$ constitutes a fuzzy comprehensive evaluation model, and then, we determine the weight, which is recorded as $W=\left\{w_{1}, w_{2}, \ldots, w_{m}\right\}$, which satisfies $\sum_{i=1}^{m} w_{i}=1$. After synthesis, $B=W R=$ $\left(b_{1}, b_{2}, \ldots, b_{m}\right)$, and after normalization, the evaluation level of the object $P$ can be determined. Finally, we synthesize the evaluation level of vector $B$.

\subsection{Evaluation Process}

4.3.1. Evaluation of Realistic Value. We recorded the dimension of the realistic value of human resources as $P_{1}$. According to Table 1, human resources in the realistic value of the corresponding indicators (factor set $U_{1}$ ) are profit margin per capita and level of human resource development. It was scored by 10 experts. The profit margins of the three companies are as follows: $\$ 11,226.77, \$ 31,156.80$, and $\$ 6,351.80$, respectively. The results of the fuzzy processing are as follows: Shougang shares (2/excellent, 2/good, 5/ moderate, $1 /$ poor, and $0 /$ very poor) (this result indicates that
2 experts think profit margin per capita is very good, 2 experts think it is good, 5 experts think it is moderate, 1 expert thinks it is poor, and no one thinks it is very poor); Baosteel shares (4/excellent, 5/good, 1/moderate, 0/poor, and 0/very poor); and Angang shares (0/very good, 4/good, $4 /$ common, 2 /poor, and $0 /$ very poor). Similarly, the evaluation results of the three companies on level of human resource development are as follows: Shougang shares (1/ excellent, 3/good, 4/moderate, 1 /poor, and 1 /very poor); Baosteel shares (3/excellent, 6/good, 1/moderate, 0/poor, 0/ very poor); and Angang shares (1/excellent, 5/good, 3/ moderate, $1 /$ poor, $0 /$ very poor).

According to $R=\left(r_{i j}\right)_{n \times m}$, the fuzzy evaluation matrix was established, i.e., $R_{1 S}=\left[\begin{array}{lllll}2 & 2 & 5 & 1 & 0 \\ 1 & 3 & 4 & 1 & 1\end{array}\right], R_{1 B}=\left[\begin{array}{lllll}4 & 5 & 1 & 0 & 0 \\ 3 & 6 & 1 & 0 & 0\end{array}\right]$, and $R_{1 A}=\left[\begin{array}{lllll}0 & 4 & 4 & 2 & 0 \\ 1 & 5 & 3 & 1 & 0\end{array}\right]$. The weight of the two indi-

cators in this dimension is $W_{1}=\left[\begin{array}{ll}0.6 & 0.4\end{array}\right]$, and the comprehensive evaluation of Shougang's human resources in this dimension is $B_{1 S}=W_{1} R_{1 s}=\left(b_{1}, b_{2}, \ldots, b_{m}\right)=\left[\begin{array}{ll}0.6 & 0.4\end{array}\right]$. $\left[\begin{array}{lllll}2 & 2 & 5 & 1 & 0 \\ 1 & 3 & 4 & 1 & 1\end{array}\right]=\left[\begin{array}{lllll}1.6 & 2.4 & 4.6 & 1 & 0.4\end{array}\right]$; after normalization, 
$B_{1 S}=\left[\begin{array}{lllll}0.16 & 0.24 & 0.46 & 0.1 & 0.04\end{array}\right]$ can be obtained, given a scale $M$, i.e., $M=\left[\begin{array}{lllll}1.0 & 0.8 & 0.6 & 0.4 & 0.2\end{array}\right]$. The fuzzy vector $B_{1 S}$ of the judgment level is synthesized, and the synthesized value $d_{1 S}=M \cdot B_{1 S}^{T}=0.676$; i.e., the realistic value of Shougang's human resources is 0.676. Similarly, the evaluation values of Baosteel shares and Angang shares can be obtained as $d_{1 B}=M \cdot B_{1 B}^{T}=0.865$ and $d_{1 A}=$ $M \cdot B_{1 A}^{T}=0.672$.

4.3.2. Evaluation of Sustainable Value. We recorded the dimension of the sustainable value of human resources as $P_{2}$. Factor set $U_{2}$ contains two indicators: the cost of low-carbon human resource management and learning level of employees. The evaluation results of the three enterprises in the previous indicator are as follows: Shougang shares $(2,3,5,0$, $0)$, Baosteel shares $(5,5,0,0,0)$, and Angang shares $(1,4,4,1$, $0)$. Similarly, the latter indicator of the three enterprises was evaluated as Shougang shares $(3,2,3,2,0)$, Baosteel shares $(5,4,1,0,0)$, and Angang shares $(2,4,3,1,0)$. According to $R=\left(r_{i j}\right)_{n \times m}$, a fuzzy evaluation matrix is established; i.e.,

$R_{2 S}=\left[\begin{array}{lllll}2 & 3 & 5 & 0 & 0 \\ 3 & 2 & 3 & 2 & 0\end{array}\right], \quad R_{2 B}=\left[\begin{array}{lllll}5 & 5 & 0 & 0 & 0 \\ 5 & 4 & 1 & 0 & 0\end{array}\right]$

and

$R_{2 A}=\left[\begin{array}{lllll}1 & 4 & 4 & 1 & 0 \\ 2 & 4 & 3 & 1 & 0\end{array}\right]$. In this dimension, the weight of the

two indicators is $W_{2}=\left[\begin{array}{ll}0.5 & 0.5\end{array}\right]$, and Shougang's evaluation on the real-value dimension is $B_{2 S}=W_{2} R_{2 s}=\left(b_{1}, b_{2}\right.$, $\left.\ldots, b_{m}\right)=\left[\begin{array}{ll}0.5 & 0.5\end{array}\right] \cdot\left[\begin{array}{lllll}2 & 3 & 5 & 0 & 0 \\ 3 & 2 & 3 & 2 & 0\end{array}\right]=\left[\begin{array}{ccccc}2.5 & 2.5 & 4 & 1 & 0\end{array}\right]$. $B_{2 S}=\left[\begin{array}{lllll}0.25 & 0.25 & 0.4 & 0.1 & 0\end{array}\right]$; given a scale $M$, fuzzy vector $B_{2 S}$ is synthesized, and we can get the synthetic value $d_{1 S}=M \cdot B_{1 S}^{T}=0.75$; that is, the evaluation value of Shougang's human resources in this dimension is 0.75 . Similarly, the evaluation values of Baosteel shares and Angang shares can be obtained as $d_{1 B}=M \cdot B_{1 B}^{T}=0.89$ and $d_{1 A}=M \cdot B_{1 A}^{T}=0.72$.

4.3.3. Evaluation of Inimitable Value. We recorded the inimitable value of human resources as $P_{3}$. $U_{3}$ is the set of factors corresponding to this dimension, and it includes two indicators: low-carbon level of human resource management and low-carbon quality index of employees. The results of expert evaluation of the previous indicator are Shougang shares $(3,3,3,1,0)$, Baosteel shares $(4,6,0,0,0)$, and Angang shares $(2,5,3,0,0)$. Similarly, the experts evaluated the latter indicator as follows: Shougang shares $(1,5,4,0,0)$, Baosteel shares $(2,6,2,0,0)$, and Angang shares $(3,6,1,0,0)$.

According to $R=\left(r_{i j}\right)_{n \times m}$, we built three fuzzy evaluation matrices, i.e., $R_{3 S}=\left[\begin{array}{lllll}3 & 3 & 3 & 1 & 0 \\ 1 & 5 & 4 & 0 & 0\end{array}\right], R_{3 B}=\left[\begin{array}{lllll}4 & 6 & 0 & 0 & 0 \\ 2 & 6 & 2 & 0 & 0\end{array}\right]$, and $R_{3 A}=\left[\begin{array}{lllll}2 & 5 & 3 & 0 & 0 \\ 3 & 6 & 1 & 0 & 0\end{array}\right]$. The weight of the two indicators is $W_{3}=\left[\begin{array}{ll}0.6 & 0.4\end{array}\right]$. The comprehensive evaluation of Shougang shares in this dimension is $B_{3 S}=\mathrm{W}_{3} R_{3 s}=\left(b_{1}, b_{2}, \ldots, b_{m}\right)=$ $\left[\begin{array}{ll}0.6 & 0.4\end{array}\right] \cdot\left[\begin{array}{lllll}3 & 3 & 3 & 1 & 0 \\ 1 & 5 & 4 & 0 & 0\end{array}\right]=\left[\begin{array}{lllll}2.2 & 3.8 & 3.4 & 0.6 & 0\end{array}\right]$. After

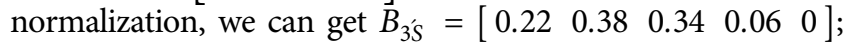
given a scale $M, d_{3 S}=M \cdot B_{3 S}^{T}=0.752$. Therefore, the evaluation value of Shougang in this dimension is 0.752. Similarly, the evaluation values of Baosteel shares and Angang shares are $d_{3 B}=M \cdot B_{3 B}^{T}=0.848$ and $d_{3 A}=M \cdot B_{3 A}^{T}=0.808$, respectively.

4.3.4. Spatial Orientation. According to the results obtained above, the evaluation value vectors of the human resources can be obtained as $S G_{1}(0.6760 .750 .752), B S_{1}$ (0.865 0.890.848), and $A S_{1}(0.6720 .720 .808)$, as shown in Figure 4.

Similarly, the position of the other 4 items in the value space can be obtained.

The value vectors of the low-carbon technology levels of the three companies in the value space are $S G_{2}(0.665$ $0.7360 .824), B S_{2}(0.9720 .90 .824)$, and $A S_{2}\left(\begin{array}{lll}0.712 & 0.808\end{array}\right.$ $0.743)$.

The evaluation value vectors of culture construction are $S_{3}\left(\begin{array}{lll}0.685 & 0.652 & 0.97\end{array}\right), B_{3}\left(\begin{array}{llll}0.868 & 0.888 & 0.81\end{array}\right)$, and $A S_{3}$ (0.836 0.8560 .79$)$.

The evaluation value vectors of market construction are $S G_{4}(0.7040 .7660 .776), B S_{4}(0.80 .8060 .86)$, and $A S_{4}(0.772$ $0.8140 .832)$.

The evaluation value vectors of management level are

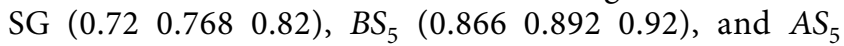
(0.834 0.848 0.78). Based on this, the structural distribution of the three enterprises can be drawn, as shown in Figure 5.

The weight of each carbon-intangible asset in Table 1 is set to $H_{i}$, and the evaluation vector of low-carbon competitiveness of the three enterprises based on carbon-intangible assets can be synthesized by using the vector sum method: 


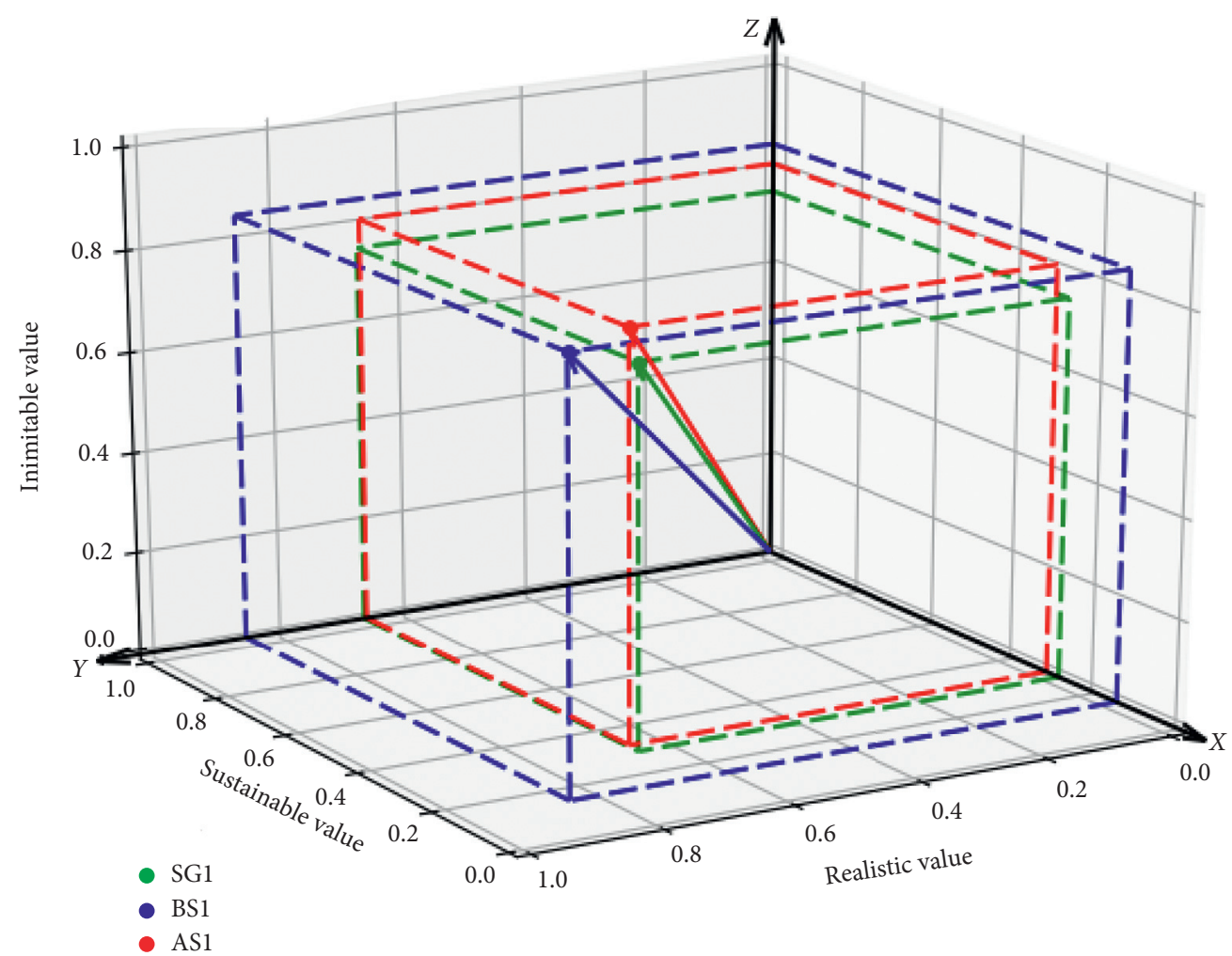

FIGURE 4: Spatial orientation of human resources of the three companies: SG (Shougang shares); BS (Baosteel shares); AS (Angang shares).

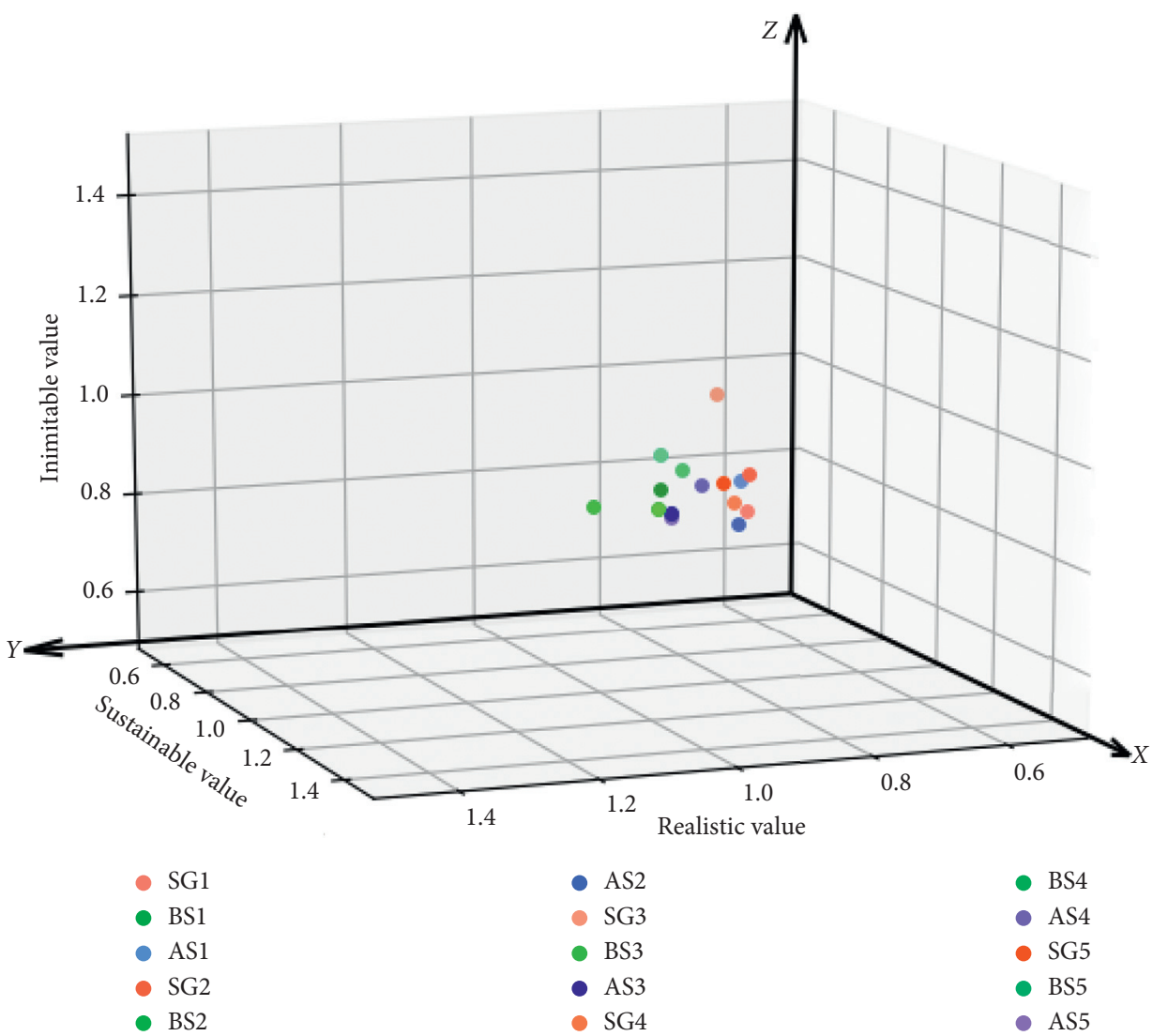

Figure 5: The value space structure of three companies: SG (Shougang shares); BS (Baosteel shares); AS (Angang shares). 


$$
\begin{aligned}
F(S G)= & H_{1} A_{1}+H_{21} A_{2}+H_{3} A_{3}+H_{4} A_{4}+H_{5} A_{5}, \\
= & 0.13 *(0.6760 .750 .752)+0.26 *(0.6650 .7360 .824)+0.16 *(0.6850 .6520 .97) \\
& +0.22 *(0.7040 .7660 .776)+0.23 *(0.720 .7680 .82)=(0.68630 .73830 .8265), \\
F(B S)= & H_{1} B_{1}+H_{2} B_{2}+H_{3} B_{3}+H_{4} B_{4}+H_{5} B_{5}, \\
= & 0.13 *(0.8650 .890 .848)+0.26 *(0.9720 .90 .824)+0.16 *(0.8680 .8880 .81) \\
& +0.22 *(0.80 .8060 .86)+0.23 *(0.8660 .8920 .92)=(0.87920 .87430 .8549), \\
F(A S)= & H_{1} C_{1}+H_{2} C_{2}+H_{3} C_{3}+H_{4} C_{4}+H_{5} C_{5}, \\
= & 0.13 *(0.6720 .720 .808)+0.26 *(0.7120 .8080 .743)+0.16 *(0.8360 .8560 .79) \\
& +0.22 *(0.7720 .8140 .832)+0.23 *(0.8340 .8480 .78)=(0.76790 .81480 .7871) .
\end{aligned}
$$

We summarize the analytical results of the above three companies in three dimensions (see Table 2 and Figure 6).

The above shows the calculation process of the actual operation of this evaluation method through a series of fuzzy mathematical methods of treatment, such that the lowcarbon competitiveness of the three enterprises can quantitatively be compared. Therefore, this evaluation method facilitates, to a certain extent, the ability to quantify and compare the abstract concept of low-carbon competitiveness.

\section{Discussion and Implications}

The evaluation of low-carbon competitiveness of enterprises is a new and broad field of research on the competitiveness of enterprises. There is limited information on this subject, and many challenges need to be addressed [5]. We selected the three dimensions of realistic value, sustainable value, and nonimitation value from the perspective of carbon-intangible assets and then constructed an evaluation space and index system of low-carbon competitiveness of enterprises; then, we selected three Chinese steel companies to carry out empirical research. First, we found that Shougang shares scored 0.6863 on the realistic value dimension compared to Angang's 0.7679. Baosteel, whose low-carbon competitiveness has the highest realistic value, scored 0.8792. Secondly, Baosteel scored 0.8743 on the sustainable value and higher than Shougang shares (0.7383) and Ansteel shares (0.8148). Finally, Angang shares scored 0.7871 on inimitable value dimension and lower than Shougang shares (0.8265) and Baosteel shares (0.8424), which shows that the ability of Angang shares in inimitable value dimension needs to be strengthened. We further traced the spatial positioning of carbon-intangible assets and their original data and found that the spatial distribution of carbon-intangible assets in Baosteel and Angang shares was quite different, while the spatial distribution and original data of Shougang shares and Angang shares were similar. It shows that the formation of low-carbon competitiveness of enterprises is complex. Thus, although the evaluation value of low-carbon competitiveness could be similar, the root causes of its formation may be very different.

Baosteel shares had higher scores in the evaluation of all kinds of carbon-intangible assets, indicating that its carbonintangible assets are balanced and its overall low-carbon competitiveness is also stable at the highest. Going forward, Baosteel shares should continue to maintain a balanced development in order to consolidate their low-carbon competitive advantage. Most of the carbon-intangible assets of Shougang shares scored relatively low in the realistic value dimension (all below 0.75), but the low-carbon market construction and management level scored higher in the sustainable value dimension (all above 0.76 ). This shows that the low-carbon market construction and management level of Shougang shares are sustainable. In addition, Shougang's low-carbon technology level, culture construction, and management level scored higher in inimitable value dimension (over 0.82), indicating that Shougang's low-carbon technology, cultural construction, and management level are difficult to imitate. The scores of various carbon-intangible assets of Ansteel shares in all dimensions were relatively stable and were no higher than 0.9 . The minimum value to achieve in terms of human carbon-intangible assets in the dimension of realistic value is 0.672 . This shows that all kinds of carbon-intangible assets of Ansteel shares are relatively stable.

With reference to the successful experience of low-carbon development in developed countries, combined with the characteristics and requirements of low-carbon economic development, we opine that the improvement of low-carbon competitiveness of steelworks should be carried out from the following aspects: first, these companies need to improve the quality of human resources. They ought to recognize the inevitability and urgency of low-carbon economy, establish the concept of low-carbon economy, actively promote the lowcarbon quality of human resources, and integrate low-carbon thinking into the introduction, development, and training of talents. Second, strengthening the development and innovation of low-carbon technology must be keenly pursued. Low-carbon technology development and innovation are the basis of lowcarbon intellectual property rights. Steel enterprises should base their operations on the cultivation of employee awareness while nurturing talents, with guaranteed investment in research and development. They must actively improve the strength of the research and development link and increase the possibility of low-carbon technological innovation. Third, enterprises must develop the concept of green manufacturing to improve their energy management systems. In the organizational structure of enterprises, functional departments such as lowcarbon and energy management must be set up, or full-time 
TABLE 2: Evaluation results of low-carbon competitiveness of three companies.

\begin{tabular}{lcccccc}
\hline & \multicolumn{2}{c}{ Realistic value } & \multicolumn{2}{c}{ Sustainable value } & \multicolumn{2}{c}{ Inimitable value } \\
& Number & Rate & Number & Rate & Number & Rate \\
\hline Shougang shares & 0.6863 & Weak & 0.7383 & Weak & 0.8265 & Medium \\
Baosteel shares & 0.8792 & Strong & 0.8743 & Strong & 0.8424 & Strong \\
Angang shares & 0.7679 & Medium & 0.8148 & Medium & 0.7871 & Weak \\
\hline
\end{tabular}

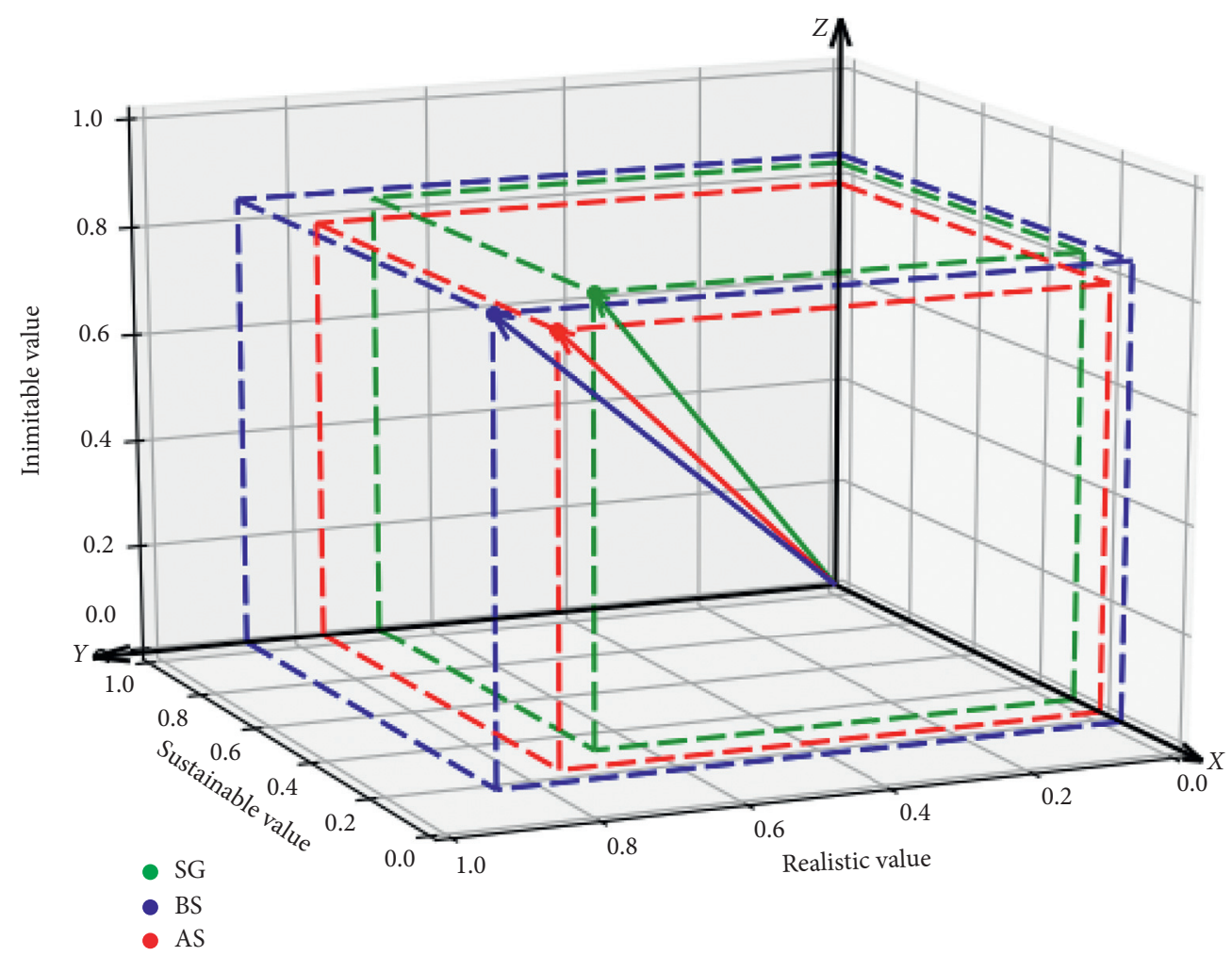

FIGURE 6: Evaluation results of low-carbon competitiveness of the companies: SG (Shougang shares); BS (Baosteel shares); AS (Angang shares).

managers of these elements must be appointed in other departments. These departments or personnel can promote the low-carbon development of enterprises with professional expertise. In addition, low-carbon management departments need to directly intervene in energy conservation or reduction in energy consumption so that enterprises can better perform in energy consumption and environmental protection. Fourth, there is a need to build low-carbon culture. Steel enterprises need to integrate low-carbon culture into their processes, production, and sales and institutionalize it to form a behavioral culture with low-carbon characteristics. Enterprises should advocate and encourage employees to form a good sense of energy conservation and environmental protection and then control or reduce the carbon emissions of individuals and their production. Last, but not least, steel enterprises must vigorously develop green marketing. Green marketing is the development and extension of marketing theory, which represents the application of a sustainable developmental view in the field of marketing. In their marketing activities, steel enterprises should conform to the requirements of the strategy of sustainable development of the times, pay attention to the protection of the ecological environment, promote the coordinated development of economy and ecological environment, and realize the harmonization of enterprise, consumer, social, and ecological environmental interests.

\section{Conclusions and Limitations}

Under the low-carbon economy, low-carbon competitiveness will become an integral part of enterprise competitiveness, and it will also be the driving force for the survival and sustainability of enterprises [26]. As a major source of industrial carbon emissions, the steel industry is pressing to reduce emissions. If enterprises do not take timely measures to improve their low-carbon competitiveness, in compliance to high-pressure government policies to limit emissions in the future, enterprises will be very passive or even defunct. Against this background, we explored the low-carbon competitiveness of steel enterprises from the perspective of carbon-intangible assets in this paper.

In order to address the challenge that makes the traditional method of evaluation inefficient, i.e., the 
multidimensional contribution of indicators [31], we proposed a systematic evaluation method. First, we constructed the value space of low-carbon competitiveness from the three dimensions of realistic, sustainable, and inimitable values and evaluated all the carbon-intangible assets of enterprises as a whole system. To compare the strengths and weaknesses of enterprises, we needed to quantify the concept of low-carbon competitiveness. Based on the analysis carried out above, this evaluation method has achieved this objective to some extent. At the same time, companies can also rely on these findings to address some shortcomings in their management of corporate carbon-intangible assets and focus on making these assets play the needed roles in the development and operation of the entire system. Compared with the traditional evaluation method, our systematic method has the following advantages: (1) the principle of the method is very simple and easy to understand, and its geometric significance is very clear. (2) This method cannot only facilitate the comparison of the contributions of different dimensions of the same indicator but also enable the comparison of different evaluation objects, thus reflecting the systematic low-carbon competitiveness of enterprises. (3) It visually and intuitively reflects the size of low-carbon competitiveness of the research object, and it is operable.

Although we have made every effort to allude to the relevant literature and conduct an in-depth research, there will always be areas that are not thoroughly covered and will need further development.

First, research on low-carbon competitiveness of enterprises is generally sparse. We do not clearly point out which carbon-intangible assets reflect the core competitiveness of enterprises in this paper. The five carbonintangible assets adopted in this paper were based on extensive literature review [17]. We also considered the views of many enterprises, which can better reflect the main content of low-carbon competitiveness of enterprises. However, it should be pointed out that the lowcarbon competitiveness of each industry is not necessarily reflected by the same group of carbon-intangible assets, and the system that affects the low-carbon competitiveness of large enterprises is not necessarily the same as that of small enterprises. Therefore, when using the evaluation method in this paper, the key carbon-intangible assets should be selected according to the actual situation of the enterprise. Second, the evaluation index system and evaluation method of a certain carbon-intangible asset in various dimensions should have scientific design standards, which need to be studied. Third, in this research, we used the vector sum method to calculate the low-carbon competitiveness of enterprises, which may yield the same vector but different distribution structures of carbonintangible assets. Therefore, a more scientific approach will be to look for the pattern between the structural shape of a firm's carbon-intangible asset distribution and its low-carbon competitiveness and then use this pattern to analyze the low-carbon competitiveness of the firm. Finally, we selected three evaluation dimensions; whether we can choose more dimensions and show them through the graph needs further study.

\section{Data Availability}

The data of this paper come from the official website of sample enterprises, which can be obtained.

\section{Conflicts of Interest}

The authors declare that they have no conflicts of interest.

\section{Acknowledgments}

This research was financially supported by the National Natural Foundation of China (Grant no. 71271177), Mineral Resources Research Center of Sichuan Province Foundation, China (Grant no. SCKCZY2020-YB009), Philosophy and Social Science Planning Project of Chengdu, China (Grant no. YY2320200701), County Economy Research Center of Sichuan Province Foundation, China (Grant no. xy2019014), and Planning Project of Sichuan Province Social Science Fund, China (Grant no. SC19TJ018).

\section{References}

[1] X. Zheng, C. Hui, and S. Yeh, "Response of ENSO amplitude to global warming in CESM large ensemble: uncertainty due to internal variability," Climate Dynamics, vol. 50, no. 11-12, pp. 4019-4035, 2018.

[2] D. D. Zhang, H. F. Lee, C. Wang et al., "Climate change and large-scale human population collapses in the pre-industrial era," Global Ecology and Biogeography, vol. 20, no. 4, pp. 520-531, 2011.

[3] N. Johnson, D. Amaya, Q. Ding, Y. Kosaka, H. Tokinaga, and S. Xie, "Multidecadal modulations of key metrics of global climate change," Global and Planetary Change, vol. 188, pp. 1-21, 2020.

[4] A. Singh, S. Unnikrishnan, M. Naik, and S. Sayanekar, "CDM implementation towards reduction of fugitive greenhouse gas emissions," Environment, Development and Sustainability, vol. 21, no. 2, pp. 569-586, 2019.

[5] Y. Jiang, J. Zhang, D. Asante, and Y. Yang, "Dynamic evaluation of low-carbon competitiveness(LCC) based on improved technique for order preference by similarity to an Ideal Solution (TOPSIS) method: a case study of Chinese steelworks," Journal of Cleaner Production, vol. 217, pp. 484-492, 2019.

[6] Y. Wang, Q. Lan, F. Jiang, and C. Chen, "Construction of China's low-carbon competitiveness evaluation system," International Journal of Climate Change Strategies and Management, vol. 12, no. 1, pp. 74-91, 2020.

[7] Z. Zhou, T. Zhang, K. Wen, H. Zeng, and X. Chen, "Carbon risk, cost of debt financing and the moderation effect of media attention: evidence from Chinese companies operating in high-carbon industries," Business Strategy and the Environment, vol. 27, no. 8, pp. 1131-1144, 2018.

[8] N. Liu, L. Fan, and X. Chen, "Key factors influencing of low carbon competitiveness," Journal of Management Modernization, vol. 37, no. 1, pp. 57-59, 2017, in Chinese.

[9] H. Yang and J. Zhang, "The strategies of advancing the cooperation satisfaction among enterprises based on low carbon supply chain management," Energy Procedia, vol. 5, no. 5, pp. 1225-1229, 2011. 
[10] X. Gao, Carbon Intangible Assets under the Perspective of Low Carbon Competitiveness Evaluation System, Southwest Jiaotong University, Chengdu, China, 2014, in Chinese.

[11] Y. Jiang, The Low-Carbon Competitiveness Evaluation Research Based on Carbon-Intangible Assets, Southwest Jiaotong University, Chengdu, China, 2016, in Chinese.

[12] G. S. Uddin, J. A. Hernandez, S. J. H. Shahzad, and A. Hedström, "Multivariate dependence and spillover effects across energy commodities and diversification potentials of carbon assets," Energy Economics, vol. 71, no. 3, pp. 35-46, 2018.

[13] L. Han and G. Huang, "Carbon asset attribute and pricing of technology," Statistical Research, vol. 32, no. 2, pp. 10-15, 2015, in Chinese.

[14] V. Frederick and A. Rezai, "The risk of policy tipping and stranded carbon assets," Journal of Environmental Economics and Management, vol. 100, no. 3, pp. 1-21, 2020.

[15] M. Li, L. Xu, and G. Chen, "A dynamic combined evaluation method based on consistency," Journal of Management Science in China, vol. 24, no. 10, pp. 149-155, 2016, in Chinese.

[16] Y. Jiang, L. Fan, Y. Yu, and G. Shi, "Research on the evaluation of carbon-intangible assets in business based on internal value network," Low Carbon Economy, vol. 5, no. 4, pp. 172-179, 2014.

[17] L. Fan and Y. Jiang, "Low carbon competitiveness evaluation of steel enterprise based on the entropy value method," Soft Science, vol. 30, no. 8, pp. 42-46, 2016, in Chinese.

[18] L. Xin, "Low carbon economic competitiveness statistic measure analysis under big data perspective," Basic \& Clinical Pharmacology \& Toxicology, vol. 125, no. 5, pp. 97-98, 2020.

[19] B. Wernerfelt, "The resource-based view of the firm: ten years after," Strategic Management Journal, vol. 16, no. 3, pp. 171-174, 1995.

[20] H. Liu and Q. Liu, "Identification path and validation of carbon-intangible assets in enterprises," Journal of Coastal Research, vol. 93, pp. 862-865, 2019.

[21] S. Cadez and A. Czerny, "Climate change mitigation strategies in carbon-intensive firms," Journal of Cleaner Production, vol. 112, no. 5, pp. 4132-4143, 2016.

[22] R. Newell and J. Zapantis, "The effects of economic and policy incentives on carbon mitigation technologies," Energy Economics, vol. 28, no. 5-6, pp. 562-578, 2006.

[23] T. F. Cojoianu, G. L. Clark, A. G. F. Hoepner, P. Veneri, and D. Wójcik, "Entrepreneurs for a low carbon world: how environmental knowledge and policy shape the creation and financing of green start-ups," Research Policy, vol. 49, no. 6, Article ID 103988, 2020.

[24] K. Hanna, E. McGuigan, B. Noble, and J. Parkins, "An analysis of the state of impact assessment research for low carbon power production: building a better understanding of information and knowledge gaps," Energy Research \& Social Science, vol. 50, pp. 116-128, 2019.

[25] T. C. Kuo, H. M. Chen, C. Y. Liu, J.-C. Tu, and T.-C. Yeh, "Applying multi-objective planning in low-carbon product design," International Journal of Precision Engineering and Manufacturing, vol. 15, no. 2, pp. 241-249, 2014.

[26] J. Xiao, Y. Shen, H. Wang, and Q. Zhou, "Estimation of industrial enterprises in low carbon competitiveness using anp and entropy methods," Natural Science Journal of Xiangtan University, vol. 7, no. 4, pp. 111-116, 2015.

[27] N. Liu and L. Fan, "The spatial value evaluation of the enterprises' carbon intangible assets from the strategic perspective," Soft Science, vol. 32, no. 2, pp. 111-116, 2020, in Chinese.
[28] X. Sun, T. Chao, S. Wang, and M. Yang, "A synthetic evaluation method based on the grey analytic hierarchy model," in Proceedings of the 33rd Chinese Control Conference, pp. 6406-6411, Nanjing, China, July 2014.

[29] D. De, S. Chowdhury, P. K. Dey, and S. K. Ghosh, "Impact of lean and sustainability oriented innovation on sustainability performance of small and medium sized enterprises: a data envelopment analysis-based framework," International Journal of Production Economics, vol. 219, pp. 416-430, 2020.

[30] Y. Jiang and L. Fan, "Influence factors of low-carbon competitiveness-empirical study based on perspective of carbonintangible assets," Technology Economics, vol. 34, no. 5, pp. 78-85, 2015, in Chinese.

[31] Y. Guo, Comprehensive Evaluation Theory and Application, Science Press, Beijing, China, 2007, in Chinese. 